\title{
Neurochemical Enhancement of Conscious Error Awareness
}

\author{
Robert Hester, ${ }^{1}$ L. Sanjay Nandam, ${ }^{2}$ Redmond G. 0'Connell, ${ }^{4}$ Joe Wagner, ${ }^{2}$ Mark Strudwick, ${ }^{3}$ Pradeep J. Nathan, \\ Jason B. Mattingley, ${ }^{2}$ and Mark A. Bellgrove ${ }^{2}$ \\ ${ }^{1}$ Department of Psychological Sciences, University of Melbourne, Melbourne, 3010, Victoria, Australia, ${ }^{2}$ Queensland Brain Institute and School of \\ Psychology and ${ }^{3}$ Centre for Advanced Imaging, University of Queensland, Brisbane, Queensland, 4072, Australia, ${ }^{4}$ School of Psychology and Trinity College \\ Institute of Neuroscience, Trinity College Dublin, Dublin 2, Ireland, ${ }^{5}$ Brain Mapping Unit, Department of Psychiatry, University of Cambridge, Cambridge, \\ CB2 2QQ, United Kingdom, and ${ }^{6}$ GlaxoSmithKline, Clinical Unit Cambridge, Cambridge, CB2 2GG, United Kingdom
}

How the brain monitors ongoing behavior for performance errors is a central question of cognitive neuroscience. Diminished awareness of performance errors limits the extent to which humans engage in corrective behavior and has been linked to loss of insight in a number of psychiatric syndromes (e.g., attention deficit hyperactivity disorder, drug addiction). These conditions share alterations in monoamine signaling that may influence the neural mechanisms underlying error processing, but our understanding of the neurochemical drivers of these processes is limited. We conducted a randomized, double-blind, placebo-controlled, cross-over design of the influence of methylphenidate, atomoxetine, and citalopram on error awareness in 27 healthy participants. The error awareness task, a go/no-go response inhibition paradigm, was administered to assess the influence of monoaminergic agents on performance errors during fMRI data acquisition. A single dose of methylphenidate, but not atomoxetine or citalopram, significantly improved the ability of healthy volunteers to consciously detect performance errors. Furthermore, this behavioral effect was associated with a strengthening of activation differences in the dorsal anterior cingulate cortex and inferior parietal lobe during the methylphenidate condition for errors made with versus without awareness. Our results have implications for the understanding of the neurochemical underpinnings of performance monitoring and for the pharmacological treatment of a range of disparate clinical conditions that are marked by poor awareness of errors.

\section{Introduction}

The neural basis of error-processing has become a key research interest in cognitive neuroscience, not only because of its relevance to human cognitive performance but also because dysfunction of self-monitoring is seen in a range of clinical conditions, such as traumatic brain injury (TBI), schizophrenia, attention deficit hyperactivity disorder (ADHD), and drug addiction (Carter et al., 2001; Kaufman et al., 2003; Rubia et al., 2005; O'Keeffe et al., 2007). In these conditions, diminished awareness of errors has been linked to clinical symptoms, such as inattention, poor insight, and perseverative behavior, which in turn are predictors of poor treatment outcome and functional recovery (Mintz et al., 2004; O'Keeffe et al., 2004). Despite the clear imperative to understand and remediate such deficits, the issue of

Received Aug. 3, 2011; revised Dec. 6, 2011; accepted Dec. 28, 2011.

Author contributions: R.H., P.J.N., and M.A.B. designed research; R.H., S.L.N., J.W., M.S., and M.A.B. performed research; S.L.N., R.G.O., M.S., and P.J.N. contributed unpublished reagents/analytic tools; R.H. and M.A.B. analyzed data; R.H., R.G.O., J.B.M., and M.A.B. wrote the paper.

This work was supported by Australian Research Council Grant DP0770337 (M.A.B.), the Australian National Health and Medical Research Council [M.A.B., R.H. (Grant 519730), P.J.N., J.B.M.), and grants from the lrish Research Council for Science Engineering and Technology (R.G.O.). We thank Drs. Bruno van Swinderen and Angela Dean for comments on this manuscript and Mykalos Byrne for assistance with figures. We also thank the Wesley Hospital Pharmacy for dispensing the drugs associated with this project.

Both L.S.N. and M.A.B. have received reimbursement from Lilly Pharmaceuticals for conference travel expenses and for speaking at conferences. P.J.N. is an employee of GlaxoSmithKline Pharmaceuticals and holds shares in the company.

Correspondence should be addressed to Dr. Mark A. Bellgrove, Queensland Brain Institute and School of Psychology, University of Queensland, Brisbane 4072, QLD, Australia. E-mail: m.bellgrove@uq.edu.au.

DOI:10.1523/JNEUROSCI.4052-11.2012

Copyright $\odot 2012$ the authors $\quad 0270-6474 / 12 / 322619-09 \$ 15.00 / 0$ awareness, and its instantiation in the brain, has been primarily overlooked.

Recent electrophysiological work has demonstrated that the early stages of error processing are preconscious and are unaffected by an individual's awareness of that error but later processing stages are only initiated if the error is consciously perceived (Nieuwenhuis et al., 2001; O'Connell et al., 2007; Steinhauser and Yeung, 2010). This distinction has implications previous studies that have shown abnormalities in error-related activity in a range of clinical groups without controlling for potential differences in error awareness. Studies that have contrasted errors that participants were or were not aware of have identified a bilateral network of frontal and parietal regions (Nieuwenhuis et al., 2001; Hester et al., 2005; Klein et al., 2007). Interestingly, this work has failed to show a predictive relationship between dorsal anterior cingulate cortex (dACC) activity and error awareness (Hester et al., 2005; Klein et al., 2007), despite this region having a central role in models of performance monitoring and its dysfunction in a range of clinical disorders that involve awareness deficits.

Current models of performance monitoring argue that a phasic decrease in midbrain dopamine (DA) provides a rewardprediction error to the dACC, which coordinates post-error adaptation of behavior (Holroyd and Coles, 2002). Other models have suggested roles for norepinephrine (NE) (Nieuwenhuis et al., 2005) and serotonin (5-HT) (Cools et al., 2008) in performance monitoring (Jocham and Ullsperger, 2009). Pharmacological manipulations of error-related brain activity have shown that enhancing catecholamines with amphetamine (de Bruijn et al., 2004; de Bruijn et al., 2006) or the $\alpha 2$ adrenoceptor antagonist 


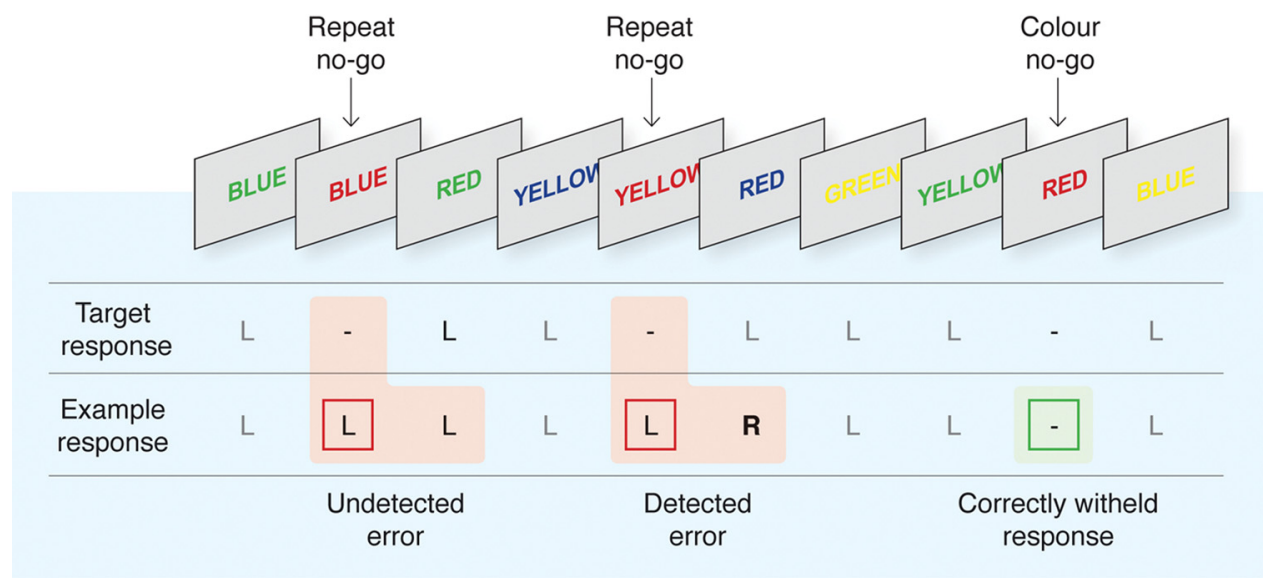

Figure 1. The EAT. The EAT presents a serial stream of single color words in incongruent fonts, with the word presented for $800 \mathrm{~ms}$ followed by a 700 ms interstimulus interval. Participants were trained to respond to each of the words with a single go trial (left) button press and to withhold this response when either of two different circumstances arose. The first was if the same word was presented on two consecutive trials (repeat no-go), and the second was if the word and font of the word matched (color no-go). To indicate error awareness, participants were trained to forego the regular go-trial button response (left; $\mathrm{L}$ ) and instead to respond with the alternative (right; $\mathrm{R})$ button after any commission error. Past studies have demonstrated that error-related BOLD signal is uninfluenced by the awareness response itself (Hester et al., 2005). Although levels of awareness undoubtedly vary on a continuum, we made a qualitative distinction between aware and unaware errors to facilitate our event-related fMRI analysis.

yohimbine (Riba et al., 2005) increased the amplitude of the error-related negativity (ERN). Methylphenidate (MPH) has also been shown to be effective in treating the cognitive deficits associated with TBI (Willmott and Ponsford, 2009), in which there is a fundamental problem of reduced awareness (O'Keeffe et al., 2004). In contrast, the $\mathrm{D}_{2}$ receptor antagonist haloperidol (Zirnheld et al., 2004) and the $\mathrm{GABA}_{\mathrm{A}}$ /benzodiazepine receptor modulators lorazepam and oxazepam (Johannes et al., 2001; de Bruijn et al., 2004) have been shown to decrease error-related dACC activity. Crucially, because the cognitive paradigms used in these studies did not explicitly test for conscious awareness of errors, one cannot disambiguate the effects of the pharmacological manipulation on preconscious versus conscious error processing.

Monoamine reuptake inhibitors, such as $\mathrm{MPH}$, atomoxetine (ATM), and citalopram (CIT), are routinely used to treat clinical disorders marked by poor error awareness. Here we examined the effect of a single acute dose of MPH, ATM, CIT, and placebo (PLAC) using a randomized, placebo-controlled, crossover design on the proportion of behavioral errors that escaped conscious awareness.

\section{Materials and Methods}

Subjects. Twenty-seven male subjects (mean age, 22 years; range, 18-35 years) were recruited for this study via advertisements at the University of Queensland (Brisbane, QLD, Australia). All participants were right handed and were excluded if they reported any history of psychiatric or neurological illness, including head injury, previous usage of psychotropic medication, or significant drug use [significant was defined as follows: (1) use of any illicit substances within the last month; (2) more than five lifetime intake of any illicit drug except cannabis; and (3) more than monthly cannabis intake], smoking (more than five cigarettes per week), or alcohol dependence ( $>24$ units per week). Before commencing, all participants were screened by a consultant psychiatrist who also administered the M.I.N.I. Screen (Sheehan et al., 1998) and the Kessler K10 (Kessler et al., 2003).

All participants were recruited according to the principles of the Declaration of Helsinki and in accordance with the ethical guidelines of the University of Queensland and the Wesley Hospital (Brisbane, QLD, Australia).

Drug administration. Participants were tested on the same day and at the same time for 4 consecutive weeks. On each occasion, the participant ingested with water (in a randomized order) a single blue gelatin capsule containing $30 \mathrm{mg}$ of MPH, $60 \mathrm{mg}$ of ATM, $30 \mathrm{mg}$ of CIT, or PLAC (dextrose). Dosage selection was based on clinically relevant doses for ADHD (Spencer et al., 1998; Michelson et al., 2003) and depression, as well as on data from previous acute challenge studies that have demonstrated neurocognitive effects of these drugs (Nathan et al., 2000; Aron et al., 2003; Chamberlain et al., 2006)

All participants were required to fast for at least $1 \mathrm{~h}$ before drug administration. Caffeine was not to be consumed on test days. After drug administration, subjects rested in a quiet waiting room for $90 \mathrm{~min}$ before commencing. From minutes +90 to +150 (after administration), subjects undertook the fMRI experiment testing error awareness. Task timing was based on the region of concomitant peak plasma levels for the three study drugs ( $\sim 90-180 \mathrm{~min}$ after oral ingestion in adults) (KraghSorensen et al., 1981; Henning and Netter, 2002; Sauer et al., 2005) and on findings from previous neurocognitive studies (Aron et al., 2003; Chamberlain et al., 2006). Data on the influence of these agents on subjective (visual analog scales) and physiological measures (e.g., blood pressure) are presented.

Behavioral task. To examine conscious recognition of errors, we administered the error awareness task (EAT) (Fig. 1) (Hester et al., 2005), a motor go/no-go response inhibition task in which subjects make errors of commission of which they are aware (aware errors) or unaware (unaware errors). Using this task, we have previously examined the neural mechanisms associated with error awareness (Hester et al., 2005; O'Connell et al., 2007), as well as the diminished awareness of performance errors in ADHD (O'Connell et al., 2009) and drug addiction (Hester et al., 2007). The EAT presents a serial stream of single color words, with the word presented for $800 \mathrm{~ms}$ followed by a $700 \mathrm{~ms}$ interstimulus interval. Participants were trained to respond to each of the words with a single "go trial" button press and withhold this response when either of two different circumstances arose. The first circumstance arose if the same word was presented on two consecutive trials ("repeat no-go") and the second if the word and its font color matched ("color no-go") rather than the typical word and color incongruency (for examples, see Fig. 1). By having two competing types of response inhibition, we aimed to vary the strength of stimulus-response relationships, whereby representations of rules competitively suppress one another such that the more prepotent rule would suppress the weaker rule and so produce a significant number of errors, a small proportion of which may go unnoticed as a result of focusing primarily on the prepotent rule. To indicate "error awareness," participants were trained to forego the regular go-trial button response (left) and instead to make a non-speeded response with the alternative (right) button on the trial immediately after a commission error. Although the online assessment of error awareness 
required that the participant make an additional overt response, control experiments with the EAT have ruled out the possibility that errorrelated brain activity might be contaminated by this additional response demand (Hester et al., 2005; O'Connell et al., 2007).

Before entering the MRI scanner, participants practiced two novel blocks of the task to ensure they understood the task instructions. Six blocks of 225 trials ( 200 go trials, 25 no-go trials) for a total of 1350 trials were administered during MRI data collection. An equivalent number of color and repeat no-go trials were administered across the six blocks for a total of 75 repeat and 75 color no-go trials. All aspects of stimulus delivery and response recording were controlled by E-Prime software (version 1.1; Psychology Software Tools) running on a laptop computer, which was interfaced with the MR scanner during acquisition of fMRI data. Participants responded to each stimulus using their right hand, entering their response on an MR-compatible response box (Fiber-Optic response pads; Current Designs).

Scanning parameters. Functional MR images were acquired at the Wesley Hospital (Auchenflower, Brisbane, QLD, Australia) using a wholebody 1.5 tesla Siemens Sonata scanner with a gradient-echo echo-planar imaging (EPI) sequence. The quadrature transmit-receive radiofrequency head coil was for signal acquisition. Echo planar images were acquired using a gradient-echo pulse sequence and sequential slice acquisition (TR, $2000 \mathrm{~ms}$; TE, $30 \mathrm{~ms}$; flip angle, $90^{\circ}$; 29 contiguous slices of $3 \mathrm{~mm}$ thickness; $10 \%$ gap; in-plane resolution of $3.6 \times 3.6$ pixels in a FOV of $384 \mathrm{~mm}$ ). A total of $174 \mathrm{EPI}$ volumes were collected for each functional run, and a total of six functional runs were performed for each participant, during each of the four sessions. Activation data were registered to high-resolution T1-weighted isotropic $\left(1 \mathrm{~mm}^{3}\right)$ structural MPRAGE images, collected during each of the four sessions, to localize the pattern of physiological changes associated with the task.

Data analysis. Behavioral data from each participant were used to categorize the no-go trial events for each drug session into successful responses (stops), aware errors, and unaware errors. All analyses were conducted using AFNI software (http://afni.nimh.nih.gov/afni/)(Cox, 1996). After image reconstruction, the time-series data were motion corrected using 3D volume registration (least-squares alignment of three translational and three rotational parameters). Activation outside the brain was removed using edge detection techniques.

Separate hemodynamic impulse response functions (IRFs) at $2 \mathrm{~s}$ temporal resolution were calculated using deconvolution techniques for aware errors, unaware errors, and stop events. Response functions for all regressor events were initiated at image acquisition onsets because the presentation of all epochs-of-interest was timed to coincide with the beginning of the $2 \mathrm{~s}$ TR cycle. A nonlinear regression program determined the best-fitting gamma-variate function for these IRFs as described previously (Murphy and Garavan, 2005). The area under the curve of the gamma-variate function was expressed as a percentage of the area under the baseline. The baseline in this design is an implicit one and is indicative of task-related go-trial processing that remains after the variance related to the other types of events have been removed.

The percentage area (event-related activation) map voxels were resampled at $1 \mathrm{~mm}^{3}$ resolution, then spatially normalized to standard MNI space (MNI 152 template), and spatially blurred with a $3 \mathrm{~mm}$ isotropic root mean squared Gaussian kernel. Group activation maps for event type (errors, stops) were determined with one-sample $t$ tests against the null hypothesis of zero event-related activation changes (i.e., no change relative to baseline). The above procedures were applied to each of the four drug sessions independently, so that group maps of errors were available for each of the four conditions. Significant voxels within group maps passed a voxelwise statistical threshold $(t=4.69, p \leq 0.00001)$ and were required to be part of a larger $72 \mu \mathrm{l}$ cluster of contiguous significant voxels. By using a combination of probability thresholding and cluster thresholding, the aim is to maximize the power of the statistical test while holding the likelihood of false positives to a minimum. To determine the cluster threshold, we use a program called $3 \mathrm{dClustSim}$. The program is provided with the number of voxels in the group map, the spatial correlation of voxels (must be contiguous on three sides), and the voxelwise threshold. The program then runs a series of Monte Carlo simulations $(10,000$ iterations for our study) to determine the frequency of clusters of

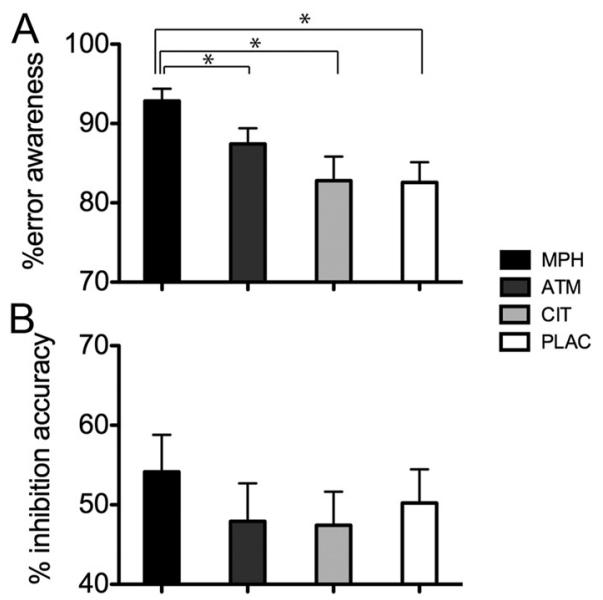

Figure 2. Behavioral performance on the EAT. $A$, The proportion of errors for which participants $(n=27)$ indicated awareness of errors as a function of the four drug conditions: MPH ATM, CIT, and PLAC. A significant main effect of drug condition was present $\left(F_{(3,78)}=10.28\right.$, $p<0.001)$. Participants were aware of a significantly higher proportion of errors in the MPH condition than in the PLAC, ATM, or CIT conditions $\left({ }^{*} p<0.008\right.$ for all comparisons, Bonferroni's adjusted). $\boldsymbol{B}$, The percentage of successful response inhibitions on EAT no-go trials during each of the four drug conditions. There was a trend toward a main effect of drug condition on the percentage of response inhibition errors $\left(F_{(3,78)}=2.32, p=0.08\right)$.

varying sizes produced by chance. From this frequency distribution, we then select the cluster size ( $72 \mu$ l given our parameters) that occurs $<1 \%$ of the time by chance, to give a threshold of $p=0.01$ (corrected).

The comparison of interest was between aware and unaware errors. Because of the significant behavioral effect of MPH on error awareness, the activation clusters from whole-brain analyses of errors in both the PLAC and MPH conditions were used to create an OR map for the purposes of a region-of-interest (ROI) analysis. An OR map includes the voxels of activation indicated as significant from either of the constituent maps. The mean activation for clusters in the combined error map was then calculated for the purposes of an ROI analysis, deriving mean activation levels for aware and unaware errors in each of the conditions, which were compared using repeated-measures $t$ tests, corrected via a modified Bonferroni's procedure for multiple comparisons (Keppel, 1991). To examine the specificity of this effect, we also performed the same analysis on the OR map of ATM and PLAC conditions.

\section{Results}

\section{Behavioral results}

Performance indices from the EAT for all four drug conditions are presented in Figure 2 and Table 1. Tests of normality revealed that behavioral measures of inhibition accuracy and error awareness from the EAT task were not normally distributed, and these were transformed using an arcsine function before analysis with parametric analyses. The proportion of EAT no-go inhibition errors of which participants $(n=27)$ indicated awareness during each of the four drug conditions (MPH, ATM, CIT, and PLAC) was examined using repeated-measures ANOVA. A significant main effect of drug condition was present $\left(F_{(3,78)}=10.28, p<\right.$ $0.001)$, with participants aware of a significantly higher proportion of errors in the MPH condition when compared with PLAC $(p<0.008$, Bonferroni's adjusted probability threshold corrected for multiple post hoc comparisons; Cohen's $d^{\prime}=$ 2.34), ATM, or CIT conditions. ATM was also associated with an improvement in error awareness compared with PLAC $(p=0.046)$, although this effect did not survive Bonferroni's adjustment.

There was a trend for response inhibition accuracy on no-go trials to differ as a function of drug condition $\left(F_{(3,78)}=2.32, p=\right.$ 
Table 1. Mean accuracy, RT, and SE of measurement scores for participants ( $n=27)$ during the four drug conditions of the EAT

\begin{tabular}{|c|c|c|c|c|c|c|c|c|}
\hline \multirow[b]{2}{*}{ Category } & \multicolumn{2}{|l|}{ Placebo } & \multicolumn{2}{|c|}{ Methylphenidate } & \multicolumn{2}{|c|}{ Atomoxetine } & \multicolumn{2}{|c|}{ Citalopram } \\
\hline & M & SEM & M & SEM & M & SEM & M & SEM \\
\hline No-go accuracy (\% correct) & 50.2 & 4.2 & 54.2 & 4.6 & 47.9 & 4.7 & 47.4 & 4.2 \\
\hline $\begin{array}{l}\text { Error awareness (\% of aware } \\
\quad \text { errors) }\end{array}$ & 80.5 & 2.4 & 91.1 & 1.9 & 85.1 & 2.1 & 81.8 & 1.9 \\
\hline Go RT (ms) & 428.5 & 18.2 & 422.7 & 19.8 & 408.6 & 21.2 & 430.2 & 20.8 \\
\hline No-go error RT (ms) & 467.2 & 23.9 & 443.8 & 20.1 & 428.4 & 21.2 & 457.5 & 25.6 \\
\hline Aware error RT (ms) & 458.1 & 22.2 & 433.4 & 20.6 & 417.2 & 23.8 & 437.6 & 25.1 \\
\hline Unaware error RT (ms) & 508.9 & 37.1 & 511.6 & 36.6 & 448.2 & 28.6 & 461.2 & 30.4 \\
\hline $\begin{array}{l}\text { Post-aware - pre-aware error RT } \\
\text { (ms) }\end{array}$ & -0.142 .6 & 16.9 & -164.4 & 17.2 & -135.4 & 18.2 & -146.6 & 17.2 \\
\hline $\begin{array}{l}\text { Post-unaware - pre-unaware } \\
\text { error RT (ms) }\end{array}$ & 36.5 & 12.2 & 23.1 & 16.5 & 95.1 & 42.3 & 57.1 & 17.1 \\
\hline $\begin{array}{l}\text { Post-aware }(n+2)-\text { pre-aware } \\
\quad \text { error RT (ms) }\end{array}$ & -88.3 & 10.2 & -84.8 & 9.6 & -86.4 & 10.7 & -88.0 & 9.3 \\
\hline
\end{tabular}

M, Mean.

0.08). Exploratory post hoc analysis demonstrated significant improvement in response inhibition in the MPH condition when compared with PLAC $\left(p=0.009\right.$, Cohen's $\left.d^{\prime}=0.40\right)$. Consistent with previous use of this task (Hester et al., 2005; O'Connell et al., 2007), individual differences in response inhibition performance were not associated with awareness of errors (PLAC, $r=-0.04$, $p=0.84 ; \mathrm{MPH}, r=0.02, p=0.91)$.

The influence of no-go type (repeat, color) on error awareness and response inhibition accuracy was also examined in separate 2 (no-go type) $\times 4$ (drug) repeated-measures ANOVAs. Error awareness rates showed a significant main effect of no-go type $\left(F_{(1,26)}=19.9, p=0.01\right)$, with poorer awareness of repeat errors compared with color, an effect previously demonstrated with this task (Hester et al., 2009). There was, however, no significant interaction between no-go type and drug condition for error awareness rates $\left(F_{(3,78)}=0.52, p=0.67\right)$. The comparable analysis for successful response inhibition demonstrated a significant main effect of no-go type $\left(F_{(1,26)}=66.6, p=0.01\right)$, with accuracy significantly higher for repeat no-go trials compared with color [also replicating a previous finding (Hester et al., 2009)] but again no interaction between no-go type and drug condition $\left(F_{(3,78)}=\right.$ 1.76, $p=0.19$ ).

There was neither a significant main effect of drug condition on go trial response time $(\mathrm{RT})\left(F_{(3,78)}=0.59, p=0.62\right)$ nor no-go error RT $\left(F_{(3,78)}=1.41, p=0.24\right)$. Although no-go error RT significantly differed between aware and unaware errors $\left(F_{(3,66)}=\right.$ $11.5, p=0.003)$, there was no interaction with drug condition $\left(F_{(3,66)}=2.24, p=0.14\right)$.

Post-error RTs showed two distinct patterns consistent with previous use of this task (Hester et al., 2005, 2007), with faster RT, or speeding up, after an aware error (post-aware error go trial $\mathrm{RT}$ - pre-aware error go trial $\mathrm{RT}=-150.6 \mathrm{~ms}$ ) and slower RT after an unaware error (post-unaware error go trial RT - preunaware error go trial RT $=52.9 \mathrm{~ms}$ ). Post-aware error RT was significantly faster than post-unaware error RT $\left(F_{(1,21)}=156.8\right.$, $p=0.01)$, but drug condition did not have a significant effect on either post-aware error RT $\left(F_{(3,78)}=1.8, p=0.15\right)$ or postunaware error $\operatorname{RT}\left(F_{(3,63)}=1.5, p=0.21\right)$. The interaction between drug condition and post-error RT changes was also nonsignificant $\left(F_{(3,63)}=0.72, p=0.54\right)$. It is important to note that the speeding of post-aware error responses is confounded by subjects providing the alternate "awareness" button press (in which the go stimulus is ignored), but a similar pattern of significantly faster RT was observed for the second go trial after an aware error.
Table 2. Regions of error-related BOLD activity in the PLAC condition map whose level of activity differentiated aware from unaware errors

\begin{tabular}{lcrrr}
\hline & & \multicolumn{3}{c}{ MNI coordinates } \\
\cline { 4 - 5 } Brain region & Volume $(\mu \mathrm{l})$ & $x$ & \multicolumn{1}{c}{$y$} & \multicolumn{1}{c}{$z$} \\
\hline Aware errors > unaware errors & & & & \\
L Inferior parietal & 3984 & -46 & -36 & 47 \\
L Anterior cingulate & 2082 & -1 & 9 & 41 \\
L Supplementary motor area & 1952 & -2 & -11 & 55 \\
R Inferior parietal & 1855 & 48 & -52 & 36 \\
L Precuneus & 1718 & -1 & -75 & 33 \\
L Insula & 332 & -53 & 10 & 8 \\
R Supramarginal & 278 & 50 & -30 & 38 \\
R Insula & 258 & 44 & 11 & 1 \\
R Inferior parietal & 191 & 38 & -42 & 42 \\
R Cerebellum & 141 & 16 & -53 & -23 \\
L Precentral & 91 & -27 & -28 & 70 \\
R Inferior frontal & 80 & 52 & 3 & 36 \\
\hline L, Left; R, right. & & & &
\end{tabular}

L, Left; $R$, right.

\section{Effects of drug on subjective ratings and blood pressure}

Participants completed visual analog scales (Norris, 1971; Bond and Lader, 1974) to assess possible drug effects on subjective alertness, contentedness, and calmness. Sixteen opposing subjective states were rated at baseline and +90 and +180 min after capsule administration. A log transformation was applied to the items. Factors of "alertness," "contentedness," and "calmness" were calculated by taking the mean transformed score across loading items for each time point. Each factor was then subject to a 4 (drug) $\times 3$ (time) repeated-measures ANOVA. Crucially, there were no significant drug $\times$ time interactions (alertness, $F_{(6,156)}=1.14, p>0.05$; contentedness, $F_{(6,156)}=0.50, p>0.05$; calmness, $\left.F_{(6,156)}=1.41, p>0.05\right)$.

Blood pressure was also recorded at baseline and then again at time points +60 and $+180 \mathrm{~min}$. There was a significant drug $\times$ time-point interaction for systolic blood pressure (drug $\times$ time, $\left.F_{(6,150)}=2.23, p<0.05\right)$. Simple effects revealed an effect of time on MPH, $(F=4.25, p=0.026)$. Post hoc tests showed that systolic blood pressure was significantly higher at +180 min $($ mean $=$ 142.62 ) relative to baseline (mean $=136.12$ ). No other simple effects were significant $(p>0.05)$. There were no significant drug $X$ time interactions for diastolic blood pressure (drug $\times$ time, $\left.F_{(6,132)}=1.18, p>0.05\right)$.

\section{Imaging data: errors}

The event-related functional analysis of BOLD activity associated with errors revealed 12 clusters of activity that differentiated aware from unaware errors (see Table 2). In contrast with null results from previous studies (Nieuwenhuis et al., 2001; Hester et al., 2005; Klein et al., 2007; O'Connell et al., 2007), we identified an activity cluster in the ACC region $(x=-1, y=9, z=41)$ that also showed significantly greater activity during aware versus unaware errors (Fig. 3). This effect appeared consistently and robustly across drug conditions, with the difference between aware and unaware error activity within this dACC ROI significant for each of the drug conditions (Fig. 3).

To examine the association between changes in BOLD activity and the behavioral improvement in awareness during the $\mathrm{MPH}$ condition, we tested for interaction effects, focusing on the comparison between MPH and PLAC in which behavioral effects were maximal. Error-related ROIs from the group maps of the PLAC and MPH conditions were combined, including all significant voxels from the two constituent maps (Hester et al., 2005), to examine mean BOLD activity during each of the four event 


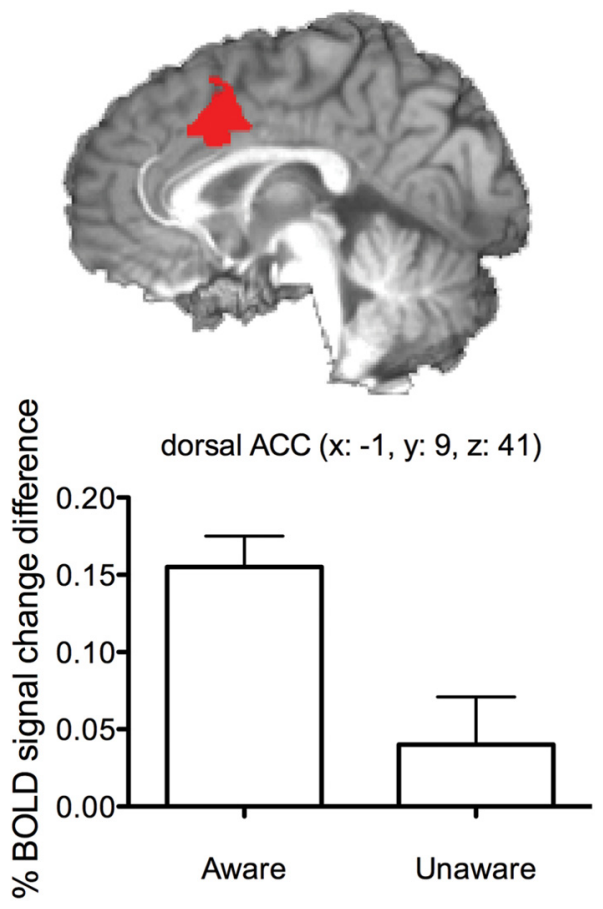

Figure 3. Activity within the $\mathrm{AACC}$ differentiates errors made with and without awareness. Bar graphs represent the mean BOLD percentage signal change for aware and unaware errors during the PLAC condition. A significant main effect of awareness on error-related ACC activity was observed during the PLAC condition, along with the three other drug conditions. The MNI coordinates for the $\mathrm{AACC}$ cluster region are listed in the title, and the sagittal 3D-rendered view of the activity cluster is taken from the MNI center-of-mass $x$-coordinate $(x=-1)$.

types. Because of the improvement in error awareness with MPH, five participants had insufficient unaware error events for inclusion in this analysis. The resulting interaction analysis was therefore based on the remaining 22 participants. We note that the benefit to error awareness conferred by MPH, relative to PLAC, remained significant in this subsample $\left(F_{(1,21)}=31.9, p<0.001\right)$ We used a two-drug condition $(\mathrm{PLAC}, \mathrm{MPH}) \times 2$ error type (aware, unaware) repeated-measures ANOVA to compare aware and unaware error-related activity during the PLAC and MPH conditions. Significant main effects for drug condition were identified in the right middle frontal, left insula, and right inferior frontal regions, with all clusters showing significantly greater activity during the MPH condition when compared with PLAC (see Table 3). Consistent with the results from the PLAC map, significant main effects of awareness were detected in bilateral inferior parietal, left insula, bilateral precuneus, bilateral middle frontal, right temporal, right cerebellar, and right angular gyrus regions. All regions showed significantly greater activity during aware errors when compared with unaware errors. The three regions showing main effects for the effect of MPH did not differentiate aware from unaware errors.

Only two clusters demonstrated significant interaction effects: the left inferior parietal lobe (IPL) and the dACC (Fig. 4). Both regions demonstrated the same pattern of activity, whereby $\mathrm{MPH}$ produced an increase in aware error activity (relative to PLAC) and a decrease in unaware error activity (relative to PLAC).

To clarify the neuroanatomical specificity of the effect of MPH on activity differences in the left IPL and dACC, we performed two additional analyses, this time comparing changes in BOLD activity and behavioral improvement in awareness during the ATM condition. First, we repeated the steps from above and substituted the ATM condition for the MPH. Significant main effects
Table 3. Regions of error-related BOLD activity in the combined PLAC and MPH OR map demonstrating significant main effects of drug condition or error awareness

\begin{tabular}{|c|c|c|c|c|}
\hline \multirow[b]{2}{*}{ Brain region } & \multirow[b]{2}{*}{ Volume $(\mu \mathrm{l})$} & \multicolumn{3}{|c|}{ MNI coordinates } \\
\hline & & $x$ & $y$ & $z$ \\
\hline \multicolumn{5}{|l|}{ Aware errors $>$ unaware errors } \\
\hline L Inferior parietal & 6569 & -43 & -39 & 51 \\
\hline R Inferior parietal & 2674 & 48 & -51 & 39 \\
\hline L Anterior cingulate & 1632 & -1 & 15 & 39 \\
\hline L Supplementary motor area & 901 & -2 & -12 & 56 \\
\hline R Middle temporal & 535 & 56 & -51 & 3 \\
\hline RPrecuneus & 437 & 5 & -74 & 33 \\
\hline R Temporal & 304 & 50 & 11 & 2 \\
\hline L Supramarginal & 221 & -56 & -26 & 25 \\
\hline L Precuneus & 203 & -8 & -77 & 32 \\
\hline L Inferior frontal & 152 & -56 & 11 & 8 \\
\hline L Inferior frontal & 145 & -57 & 1 & 11 \\
\hline R Cerebellar & 144 & 19 & -54 & -24 \\
\hline R Angular & 132 & 52 & -55 & 21 \\
\hline \multicolumn{5}{|l|}{ MPH $>$ placebo } \\
\hline R Middle frontal & 438 & 27 & 42 & 34 \\
\hline L Insula & 172 & -35 & 16 & -4 \\
\hline R Inferior frontal & 142 & 52 & 8 & 16 \\
\hline
\end{tabular}

$L$, Left; $R$, right.

for drug condition were identified in six regions, the right inferior parietal, left insula, left caudate, posterior cingulate, left supramarginal, and right middle frontal, with all clusters showing significantly greater activity during the ATM condition when compared with PLAC (Table 4). Consistent with the results from the PLAC map, significant main effects of awareness were detected in bilateral inferior parietal, left insula, bilateral precuneus, bilateral middle frontal, right temporal, right cerebellar, and thalamic regions. All regions showed significantly greater activity during aware errors when compared with unaware errors.

Two regions (Fig. 4C,D), the left inferior frontal gyrus (IFG) (MNI: $x=-54, y=3, z=13$ ) and right inferior temporal gyrus (ITG) $(x=56, y=-54, z=-6)$, demonstrated significant interaction effects. Both regions showed the same pattern of activity, whereby ATM was associated with a large increase in aware error activity (relative to PLAC) and a small decrease in unaware error activity (relative to PLAC).

Second, we used the ROIs from the PLAC condition group map to compare activity in a $2 \times 3$ repeated-measures ANOVA for awareness-related changes in BOLD activity as a function of the three drug conditions (PLAC, ATM, MPH). Three regions showed a significant interaction effect (Fig. 5), two in closely aligned regions to those identified in the PLAC and MPH comparison, namely the left IPL (MNI: $x=-45, y=-36, z=47$ ) and dACC (MNI: $x=-1, y=9, z=41$ ), as well as the right IPL (MNI: $x=48, y=-52, z=36$ ). The difference in BOLD activity between aware and unaware errors was significantly greater in the MPH condition than PLAC ( $p=0.047$, corrected $)$, whereas ATM was not significantly different from PLAC $(p=0.86)$.

Our results therefore suggest a neuroanatomical dissociation in terms of the effect of MPH and ATM on awareness-related brain activity. Whereas robust enhancement of error awareness by MPH was mediated by activity differences within the dACC and left IPL, the relatively more subtle effects of ATM were mediated by activity differences within the left IFG and right ITG.

\section{Discussion}

The results of this study demonstrate the pharmacological enhancement of conscious error awareness. A single, clinically relevant dose of MPH was able to considerably (10\%) improve error 
A

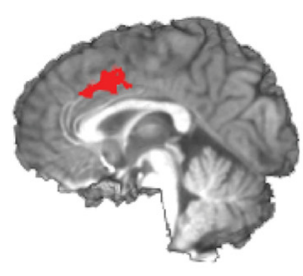

B

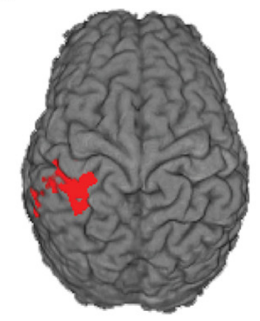

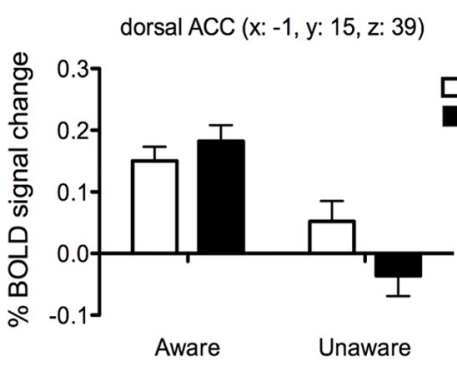

L IPL (x: $-44, y:-39, z: 51)$

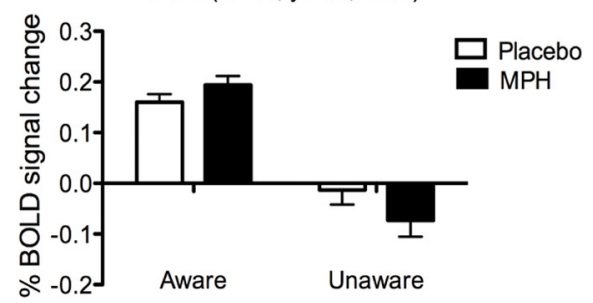

C

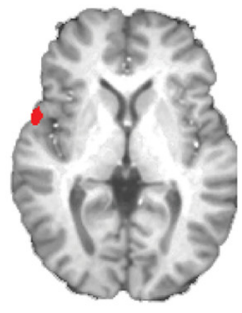

D

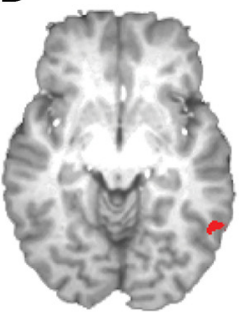

L IFG (x: -54, y: 3, z: 13)

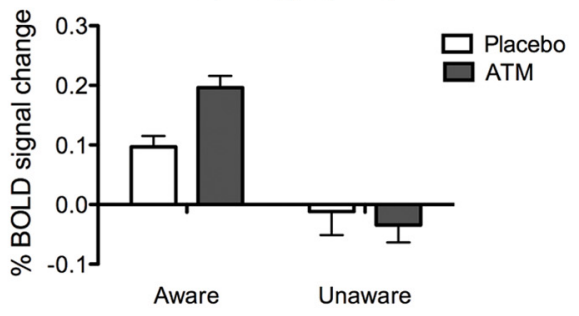

R ITG (x: 56, y: -54, z: -6)

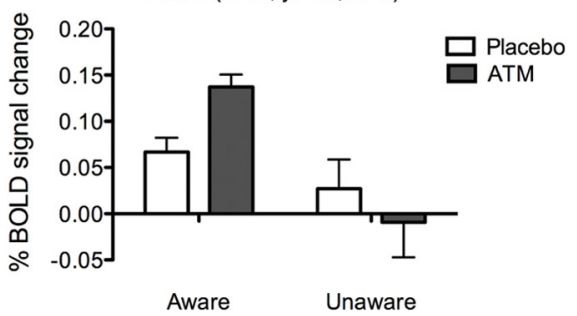

Figure 4. BOLD activity clusters for aware versus unaware errors demonstrating anatomically dissociable effects of MPH and ATM. The dACC activity cluster $(\boldsymbol{A})$ (MNI coordinates: $x=-1, y=$ $15, z=39$ ) and left IPL cluster $(\boldsymbol{B})$ (MNI coordinates: $x=-44, y=-39, z=51$ ) demonstrating a significant interaction effect between error awareness (aware, unaware) and drug condition (MPH, PLAC). The left IFG (C) (MNI coordinates: $x=-54, y=3, z=13$ ) and right ITG (D) (MNI coordinates: $x=56, y=-54, z=-6)$ represent the regions showing a significant interaction between ATM and error awareness. Bar graphs represent the mean BOLD percentage signal change (relative to baseline) for aware and unaware errors, for the MPH, ATM, or PLAC conditions. Error bars represent the SEM. L, Left; R, right.

Table 4. Regions of error-related BOLD activity in the combined PLAC and ATM OR map demonstrating significant main effects of drug condition or error awareness

\begin{tabular}{|c|c|c|c|c|}
\hline \multirow[b]{2}{*}{ Brain region } & \multirow[b]{2}{*}{ Volume ( $\mu \mathrm{l})$} & \multicolumn{3}{|c|}{ MNI coordinates } \\
\hline & & $x$ & $y$ & $Z$ \\
\hline \multicolumn{5}{|l|}{ Aware errors $>$ unaware errors } \\
\hline L Postcentral & 11716 & -41 & -33 & 50 \\
\hline R Inferior parietal & 4615 & 46 & -50 & 39 \\
\hline L Middle cingulate & 4266 & -2 & -2 & 45 \\
\hline L Precuneus & 1846 & 1 & -72 & 39 \\
\hline $\mathrm{R}$ Inferior frontal & 1667 & 43 & 8 & 8 \\
\hline R Cerebellum & 1492 & 26 & -53 & -22 \\
\hline R Thalamus & 1359 & 8 & -15 & 14 \\
\hline L Inferior frontal & 909 & -54 & 3 & 12 \\
\hline L Thalamus & 880 & -12 & -21 & 9 \\
\hline R Inferior temporal & 849 & 55 & -52 & -2 \\
\hline L Insula & 759 & -38 & 13 & 5 \\
\hline R Superior frontal & 663 & 21 & -13 & 60 \\
\hline L Cerebellum & 371 & -35 & -46 & -24 \\
\hline L Caudate & 316 & -13 & -5 & 16 \\
\hline R Posterior cingulate & 279 & 2 & -32 & 26 \\
\hline L Supramarginal & 251 & -56 & -50 & 27 \\
\hline R Middle frontal & 136 & 33 & 27 & 37 \\
\hline R Middle frontal & 116 & 41 & 28 & 31 \\
\hline R Supplementary motor area & 108 & 10 & -10 & 68 \\
\hline \multicolumn{5}{|l|}{ ATM $>$ placebo } \\
\hline R Inferior parietal & 4615 & 46 & -50 & 39 \\
\hline L Insula & 759 & -38 & 13 & 5 \\
\hline L Caudate & 316 & -13 & -5 & 16 \\
\hline R Posterior cingulate & 279 & 2 & -32 & 26 \\
\hline L Supramarginal & 251 & -56 & -50 & 27 \\
\hline R Supplementary motor area & 108 & 10 & -10 & 68 \\
\hline
\end{tabular}

awareness in nonclinical adults compared with ATM, CIT, and PLAC. The beneficial effect of MPH was specific to error awareness, without concomitant changes in response speed. Crucially, this behavioral improvement in error awareness was underpinned by physiological changes within the dACC and IPL, with activity differences between aware and unaware trials being significantly larger under MPH than under PLAC.

Holroyd and Coles (2002) have argued that performance monitoring is dependent on ascending mesencephalic DA projections that transmit predictive error signals via the basal ganglia to the ACC. The results of the current study are broadly consistent with this hypothesis, because the indirect DA agonist MPH, which has been shown to increase DA levels within subcortical regions, including the striatum and basal ganglia (Volkow et al., 2005), was able to modulate activity within the dACC and promote conscious error awareness.

Although MPH is often viewed as an exclusive DA reuptake inhibitor, in common with ATM it has profound effects on NE signaling in prefrontal cortex (PFC) via reuptake inhibition of the $\mathrm{NE}$ transporter (Berridge et al., 2006). A potential role for NE in error processing is indicated by the strong reciprocal connections between the PFC, ACC, and the locus ceruleus (LC) arousal system. The $\mathrm{LC}$ also provides a strong phasic response to transient decreases in performance and serves to boost task-specific representations in top-down control regions (Aston-Jones and Cohen, 2005; Nieuwenhuis et al., 2005). Intracranial recordings in monkeys have demonstrated that the magnitude of phasic NE cell firing is determined by tonic activity levels in the LC (Aston-Jones and Cohen, 2005), and important relationships between error awareness and arousal have been reported previously. For example, awareness of errors on the EAT is reduced by task manipulations that engender attentional drift (Shalgi et al., 2007) and is inversely correlated with tonic EEG measures of cortical arousal (O'Connell et al., 2007). These models further propose that the balance between tonic and phasic modes of LC/NE activity is driven by a representation of current task utility that may be DA dependent (Aston-Jones and Cohen, 2005). Thus, the beneficial effect of MPH, and intermediate effect of ATM, on error awareness is consistent with the view that DA and NE are likely to play overlapping, complimentary roles in the processing of errors (Jocham and Ullsperger, 2009). 
A

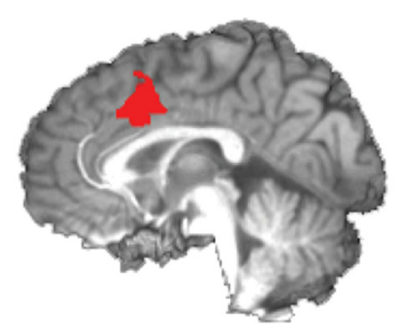

B

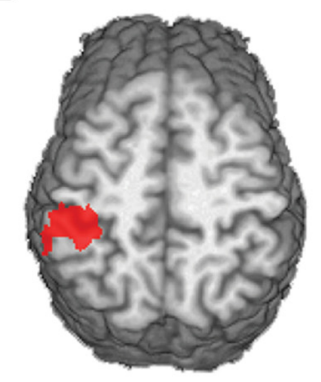

C

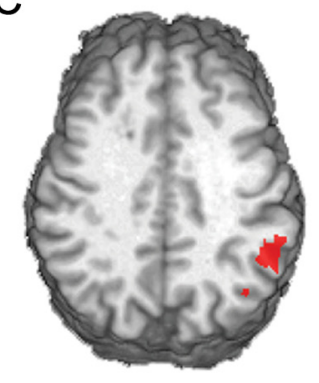

dorsal ACC (x: $-1, y: 9, z: 41)$

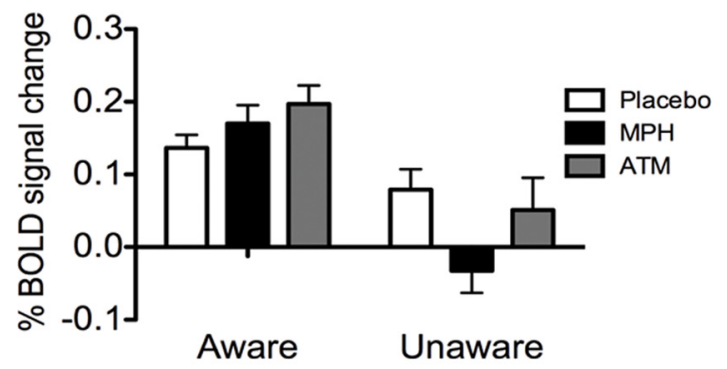

L IPL (x: $-45, y:-36, z: 47)$

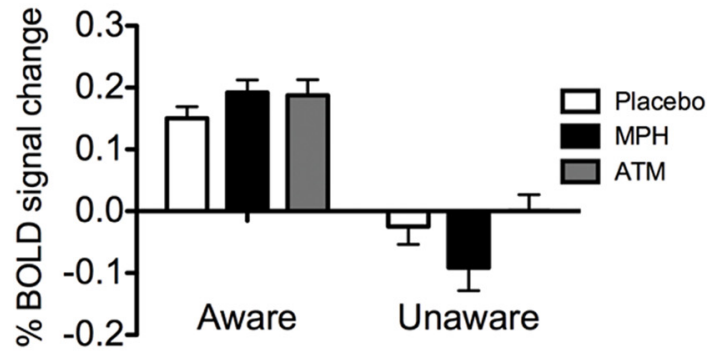

R IPL (x: 48, y: -52, z: 36)

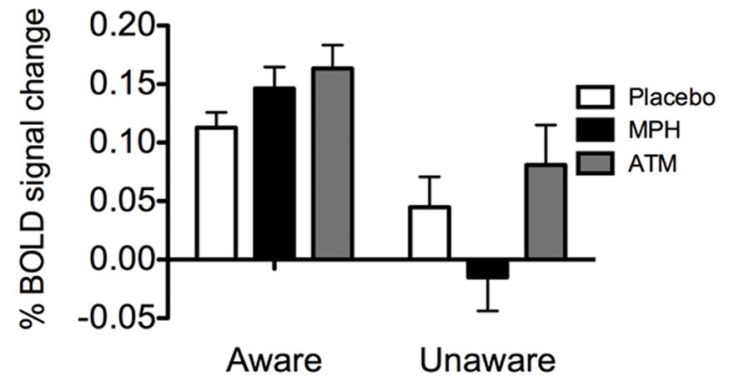

Figure 5. BOLD activity clusters from the PLAC condition map showing significant error awareness $X$ drug condition interaction effects. The dACC activity cluster ( $A$ ) (MNI coordinates: $x=-1, y=9, z=41$ ) and left IPL cluster ( $\boldsymbol{B})$ (MNI coordinates: $x=$ $-45, y=-36, z=47)$ and right IPL (C) (MNI coordinates: $x=48, y=-52, z=36$ ) demonstrating a significant interaction effect between error awareness (aware, unaware) and drug condition (MPH, ATM, PLAC). Bar graphs represent the mean BOLD percentage signal change (relative to baseline) for aware and unaware errors, for the MPH, ATM, or PLAC conditions. Error bars represent the SEM. L, Left; $R$, right.

A possible noradrenergic influence on error processing is also supported by the nominally significant effect of ATM compared with PLAC. ATM also strengthened awareness-related brain activity but within a distinct set of awareness-related regions to those targeted by MPH. The enhancement of IFG activity by ATM is consistent with known NE inputs and expression of the NE transporter within PFC (Arnsten, 2011). Our result is also consistent with a previous study that examined the impact of an acute dose of ATM ( $80 \mathrm{mg}$ ) versus PLAC on error monitoring and reported drug-related activity changes in the IFG (Graf et al., 2011). Together, our study suggests dissociable neural pathways by which MPH and ATM may improve conscious error awareness. Notwithstanding evidence that MPH modulates catecholamine signaling in PFC, the functional dissociations underpinning error awareness reported here recapitulate the known expression patterns of the molecular targets of MPH and ATM. Thus, MPH inhibits the action of DA transporter within the mesencephalic DA system projecting to the ACC, whereas ATM inhibits the action of NE transporter within prefrontal but not subcortical regions (Bymaster et al.,
2002). Future studies should assess the potential clinical utility of these findings.

The results of the current study show that enhancing catecholamine neurotransmission with $\mathrm{MPH}$ produces a more robust error signal within the ACC and inferior parietal cortex and that this same enhancement significantly increases the likelihood that errors will be consciously perceived. The specificity and size of the MPH effect on error awareness in healthy adults is directly relevant to the treatment of performance monitoring and insight deficits identified in clinical conditions. For example, MPH is routinely used to treat ADHD and has been more recently trialed as a treatment for cocaine dependence (Castells et al., 2007). These groups are known to demonstrate impaired error awareness (Hester et al., 2007; O'Connell et al., 2009) and diminished error-related neural activity in regions including the dACC (Kaufman et al., 2003; Rubia et al., 2005), with the level of impairment associated with increased symptom severity (Moeller et al., 2010). Recent evidence suggests that MPH significantly increases ACC activity in ADHD and cocaine dependence (Jonkman et al., 2007; Goldstein et al., 2010; Li et al., 2010). It is not known, however, whether chronic use of MPH during typical treatment regimens has beneficial effects for error awareness or clinical symptomatology. Given the relationship between the severity of awareness deficits and clinical symptoms in psychiatric conditions, the current data highlight the potential for such medications to enhance error awareness and in turn improve clinical outcomes.

The absence of any significant influence of CIT on behavioral or imaging measures of error processing is consistent with previous pharmacologic studies examining the acute effect of other drugs thought to influence the serotonergic system (de Bruijn et al., 2004, 2006). Previous studies using acute tryptophan depletion to perturb the serotonergic system had found a negative impact on dorsomedial PFC during cognitive control tasks, in both humans (Evers et al., 2006) and rats (Narayanan and Laubach, 2008), potentially implicating this system in performance monitoring. However, the consistent absence of influence from serotonergic manipulation on error processing in previous studies, including those examining feedback processing in the absence of punishment (Barnes et al., 2011; Cools et al., 2011), appears to discount a specific role for 5-HT in error processing.

With respect to the role of dACC in awareness of errors, we note that the finding of significantly greater dACC activity for aware errors compared with unaware errors is inconsistent with previous fMRI studies of error awareness (Hester et al., 2005, 2009; Klein et al., 2007). This finding is significant because theoretical models have consistently implicated ACC activity in performance monitoring, yet previous fMRI studies have failed to 
demonstrate a critical role for this region in conscious error detection. ERP studies of error awareness have provided mixed results with regard to the relationship between the ERN and error awareness (Scheffers and Coles, 2000; Nieuwenhuis et al., 2001; Endrass et al., 2007; O'Connell et al., 2007; Steinhauser and Yeung, 2010), with a recent review from Wessel (2012) highlighting several factors that may explain these contrary findings. One of those factors, statistical power, may in part account for our contrary finding, with the current study being the largest yet to test the association of error awareness and dACC activity. Previous fMRI studies demonstrated a nonsignificant trend toward greater dACC activity during aware errors when compared with unaware (Hester et al., 2005; Klein et al., 2007) and had effect sizes (Hester et al., 2005, Cohen's $d^{\prime}=0.32$ ) that would have more probably been significant with the sample size of the current study.

One hypothesis to account for the difference in dACC activity between aware and unaware errors is the potential for greater response conflict during aware error trials, for example, between the delayed, but unsuccessful, inhibitory control response and the subsequent error detection (or awareness) response (Nieuwenhuis et al., 2003). We have previously attempted to examine the effect of the awareness response on BOLD activity by replicating the stimuli and timing from the EAT task and requiring participants to make the awareness response after a high response conflict trial. BOLD activity during these trials indicated significant activity in only one functionally defined error-related ROI, the left middle temporal gyrus, suggesting that this response requirement does not account for the dACC difference (Hester et al., 2005). However, although this control condition attempted to capitalize on the overlearned response to the word "STOP," it did not require the participant to withhold their response. Future research could test whether this response requirement accounted for the increased dACC activity for aware errors identified by go/no-go paradigms, by temporally dissociating the awareness response from the no-go error event.

Human performance is inherently error prone, and, in some cases, action errors occur without conscious awareness. This study provides foundational evidence that the explicit awareness of errors is dependent on activity within an error-monitoring network, including the dACC, IPL, as well as frontal and cerebellar regions. Critically, we show that the catecholamine reuptake inhibitor MPH promotes the conscious awareness of performance errors by strengthening activation differences within the dACC and IPL for errors made with versus without awareness compared with PLAC. Modulation of error awareness by the NE reuptake inhibitor ATM was smaller in effect size and mediated via anatomically distinct regions, including the left IFG. Conversely, the selective 5-HT reuptake inhibitor CIT had no significant behavioral effects, suggesting that error awareness is particularly dependent on catecholaminergic neurotransmission. Our results have implications for our understanding of the neurochemical underpinnings of performance monitoring and for the pharmacological treatment of a range of disparate clinical conditions that are marked by poor awareness of errors.

\section{References}

Arnsten AF (2011) Catecholamine influences on dorsolateral prefrontal cortical networks. Biol Psychiatry 69:e89-e99.

Aron AR, Dowson JH, Sahakian BJ, Robbins TW (2003) Methylphenidate improves response inhibition in adults with attention-deficit/hyperactivity disorder. Biol Psychiatry 54:1465-1468.

Aston-Jones G, Cohen JD (2005) An integrative theory of locus coeruleus- norepinephrine function: adaptive gain and optimal performance. Annu Rev Neurosci 28:403-450.

Barnes JJ, Dean AJ, Nandam LS, O'Connell RG, Bellgrove MA (2011) The molecular genetics of executive function: role of monoamine system genes. Biol Psychiatry 69:e127-e143.

Berridge CW, Devilbiss DM, Andrzejewski ME, Arnsten AF, Kelley AE, Schmeichel B, Hamilton C, Spencer RC (2006) Methylphenidate preferentially increases catecholamine neurotransmission within the prefrontal cortex at low doses that enhance cognitive function. Biol Psychiatry 60:1111-1120.

Bond A, Lader M (1974) The use of analogue scales in rating subjective feelings. Br J Med Psychol 47:211-218.

Bymaster FP, Katner JS, Nelson DL, Hemrick-Luecke SK, Threlkeld PG, Heiligenstein JH, Morin SM, Gehlert DR, Perry KW (2002) Atomoxetine increases extracellular levels of norepinephrine and dopamine in prefrontal cortex of rat: a potential mechanism for efficacy in attention deficit/ hyperactivity disorder. Neuropsychopharmacology 27:699-711.

Carter CS, MacDonald AW 3rd, Ross LL, Stenger VA (2001) Anterior cingulate cortex activity and impaired self-monitoring of performance in patients with schizophrenia: an event-related fMRI study. Am J Psychiatry 158:1423-1428.

Castells X, Casas M, Vidal X, Bosch R, Roncero C, Ramos-Quiroga JA, Capell à D (2007) Efficacy of central nervous system stimulant treatment for cocaine dependence: a systematic review and meta-analysis of randomized controlled clinical trials. Addiction 102:1871-1887.

Chamberlain SR, Müller U, Blackwell AD, Clark L, Robbins TW, Sahakian B] (2006) Neurochemical modulation of response inhibition and probabilistic learning in humans. Science 311:861-863.

Cools R, Roberts AC, Robbins TW (2008) Serotoninergic regulation of emotional and behavioural control processes. Trends Cogn Sci 12:31-40.

Cools R, Nakamura K, Daw ND (2011) Serotonin and dopamine: unifying affective, activational, and decision functions. Neuropsychopharmacology 36:98-113.

Cox RW (1996) AFNI: software for analysis and visualization of functional magnetic resonance neuroimages. Comput Biomed Res 29:162-173.

de Bruijn ER, Hulstijn W, Verkes RJ, Ruigt GS, Sabbe BG (2004) Druginduced stimulation and suppression of action monitoring in healthy volunteers. Psychopharmacology (Berl) 177:151-160.

de Bruijn ER, Sabbe BG, Hulstijn W, Ruigt GS, Verkes RJ (2006) Effects of antipsychotic and antidepressant drugs on action monitoring in healthy volunteers. Brain Res 1105:122-129.

Endrass T, Reuter B, Kathmann N (2007) ERP correlates of conscious error recognition: aware and unaware errors in an antisaccade task. Eur J Neurosci 26:1714-1720.

Evers EA, van der Veen FM, van Deursen JA, Schmitt JA, Deutz NE, Jolles J (2006) The effect of acute tryptophan depletion on the BOLD response during performance monitoring and response inhibition in healthy male volunteers. Psychopharmacology (Berl) 187:200-208.

Goldstein RZ, Woicik PA, Maloney T, Tomasi D, Alia-Klein N, Shan J, Honorio J, Samaras D, Wang R, Telang F, Wang GJ, Volkow ND (2010) Oral methylphenidate normalizes cingulate activity in cocaine addiction during a salient cognitive task. Proc Natl Acad Sci U S A 107:16667-16672.

Graf H, Abler B, Freudenmann R, Beschoner P, Schaeffeler E, Spitzer M, Schwab M, Grön G (2011) Neural correlates of error monitoring modulated by atomoxetine in healthy volunteers. Biol Psychiatry 69:890-897.

Henning J, Netter P (2002) Oral application of citalopram (20 mg) and its usefulness for neuroendocrine challenge tests. Int J Neuropsychopharmacol 5:67-71.

Hester R, Foxe JJ, Molholm S, Shpaner M, Garavan H (2005) Neural mechanisms involved in error processing: a comparison of errors made with and without awareness. Neuroimage 27:602-608.

Hester R, Simões-Franklin C, Garavan H (2007) Post-error behavior in active cocaine users: poor awareness of errors in the presence of intact performance adjustments. Neuropsychopharmacology 32:1974-1984.

Hester R, Nestor L, Garavan H (2009) Impaired error awareness and anterior cingulate cortex hypoactivity in chronic cannabis users. Neuropsychopharmacology 34:2450-2458.

Holroyd CB, Coles MG (2002) The neural basis of human error processing: reinforcement learning, dopamine, and the error-related negativity. Psychol Rev 109:679-709.

Jocham G, Ullsperger M (2009) Neuropharmacology of performance monitoring. Neurosci Biobehav Rev 33:48-60. 
Johannes S, Wieringa BM, Nager W, Dengler R, Munte TF (2001) Oxazepam alters action monitoring. Psychopharmacology (Berl) 155:100-106.

Jonkman LM, van Melis JJ, Kemner C, Markus CR (2007) Methylphenidate improves deficient error evaluation in children with ADHD: an eventrelated brain potential study. Biol Psychol 76:217-229.

Kaufman JN, Ross TJ, Stein EA, Garavan H (2003) Cingulate hypoactivity in cocaine users during a GO/NOGO task as revealed by event-related fMRI. J Neurosci 23:7839-7843.

Keppel G (1991) Design and analysis: a researcher's handbook. Englewood Cliffs, NJ: Prentice Hall.

Kessler RC, Barker PR, Colpe LJ, Epstein JF, Gfroerer JC, Hiripi E, Howes MJ, Normand SL, Manderscheid RW, Walters EE, Zaslavsky AM (2003) Screening for serious mental illness in the general population. Arch Gen Psychiatry 60:184-189.

Klein TA, Endrass T, Kathmann N, Neumann J, von Cramon DY, Ullsperger M (2007) Neural correlates of error awareness. Neuroimage 34:1774-1781.

Kragh-Sorensen P, Overo KF, Petersen OL, Jensen K, Parnas W (1981) The kinetics of citalopram: single and multiple dose studies in man. Acta Pharmacol Toxicol (Copenh) 48:53-60.

Li CS, Morgan PT, Matuskey D, Abdelghany O, Luo X, Chang JL, Rounsaville BJ, Ding YS, Malison RT (2010) Biological markers of the effects of intravenous methylphenidate on improving inhibitory control in cocainedependent patients. Proc Natl Acad Sci U S A 107:14455-14459.

Michelson D, Adler L, Spencer T, Reimherr FW, West SA, Allen AJ, Kelsey D, Wernicke J, Dietrich A, Milton D (2003) Atomoxetine in adults with ADHD: two randomized, placebo-controlled studies. Biol Psychiatry 53:112-120.

Mintz AR, Addington J, Addington D (2004) Insight in early psychosis: a 1-year follow-up. Schizophr Res 67:213-217.

Moeller SJ, Maloney T, Parvaz MA, Alia-Klein N, Woicik PA, Telang F, Wang GJ, Volkow ND, Goldstein RZ (2010) Impaired insight in cocaine addiction: laboratory evidence and effects on cocaine-seeking behaviour. Brain 133:1484-1493.

Murphy K, Garavan H (2005) Deriving the optimal number of events for an event-related fMRI study based on the spatial extent of activation. Neuroimage 27:771-777.

Narayanan NS, Laubach M (2008) Neuronal correlates of post-error slowing in the rat dorsomedial prefrontal cortex. J Neurophysiol 100:520-525.

Nathan PJ, Sitaram G, Stough C, Silberstein RB, Sali A (2000) Serotonin, noradrenaline and cognitive function: a preliminary investigation of the acute pharmacodynamic effects of a serotonin versus a serotonin and noradrenaline reuptake inhibitor. Behav Pharmacol 11:639-642.

Nieuwenhuis S, Ridderinkhof KR, Blom J, Band GP, Kok A (2001) Errorrelated brain potentials are differentially related to awareness of response errors: evidence from an antisaccade task. Psychophysiology 38:752-760.

Nieuwenhuis S, Yeung N, van den Wildenberg W, Ridderinkhof KR (2003) Electrophysiological correlates of anterior cingulate function in a go/ no-go task: effects of response conflict and trial type frequency. Cogn Affect Behav Neurosci 3:17-26.

Nieuwenhuis S, Aston-Jones G, Cohen JD (2005) Decision making, the P3, and the locus coeruleus-norepinephrine system. Psychol Bull 131:510-532.

Norris H (1971) The action of sedatives on brain stem oculomotor systems in man. Neuropharmacology 10:181-191.
O’Connell RG, Dockree PM, Bellgrove MA, Kelly SP, Hester R, Garavan H, Robertson IH, Foxe JJ (2007) The role of cingulate cortex in the detection of errors with and without awareness: a high-density electrical mapping study. Eur J Neurosci 25:2571-2579.

O’Connell RG, Bellgrove MA, Dockree PM, Lau A, Hester R, Garavan H, Fitzgerald M, Foxe JJ, Robertson IH (2009) The neural correlates of deficient error awareness in attention-deficit hyperactivity disorder (ADHD). Neuropsychologia 47:1149-1159.

O'Keeffe FM, Dockree PM, Robertson IH (2004) Poor insight in traumatic brain injury mediated by impaired error processing? Evidence from electrodermal activity. Brain Res Cogn Brain Res 22:101-112.

O’Keeffe F, Dockree P, Moloney P, Carton S, Robertson IH (2007) Awareness of deficits in traumatic brain injury: a multidimensional approach to assessing metacognitive knowledge and online-awareness. J Int Neuropsychol Soc 13:38-49.

Riba J, Rodríguez-Fornells A, Morte A, Münte TF, Barbanoj MJ (2005) Noradrenergic stimulation enhances human action monitoring. J Neurosci 25:4370-4374.

Rubia K, Smith AB, Brammer MJ, Toone B, Taylor E (2005) Abnormal brain activation during inhibition and error detection in medicationnaive adolescents with ADHD. Am J Psychiatry 162:1067-1075.

Sauer JM, Ring BJ, Witcher JW (2005) Clinical pharmacokinetics of atomoxetine. Clin Pharmacokinet 44:571-590.

Scheffers MK, Coles MG (2000) Performance monitoring in a confusing world: error-related brain activity, judgments of response accuracy, and types of errors. J Exp Psychol Hum Percept Perform 26:141-151.

Shalgi S, O'Connell RG, Deouell LY, Robertson IH (2007) Absent minded but accurate: delaying responses increases accuracy but decreases error awareness. Exp Brain Res 182:119-124.

Sheehan DV, Lecrubier Y, Sheehan KH, Amorim P, Janavs J, Weiller E, Hergueta T, Baker R, Dunbar GC (1998) The mini-international neuropsychiatric interview (M.I.N.I.): the development and validation of a structured diagnostic psychiatric interview for DSM-IV and ICD-10. J Clin Psychiatry 59 [Suppl 20]:22-33; quiz 34-57.

Spencer T, Biederman J, Wilens T, Prince J, Hatch M, Jones J, Harding M, Faraone SV, Seidman L (1998) Effectiveness and tolerability of tomoxetine in adults with attention deficit hyperactivity disorder. Am J Psychiatry 155:693-695.

Steinhauser M, Yeung N (2010) Decision processes in human performance monitoring. J Neurosci 30:15643-15653.

Volkow ND, Wang GJ, Fowler JS, Ding YS (2005) Imaging the effects of methylphenidate on brain dopamine: new model on its therapeutic actions for attention-deficit/hyperactivity disorder. Biol Psychiatry 57:1410-1415.

Wessel JR (2012) Error awareness and the error-related negativity: evaluating the first decade of evidence. Front Hum Neurosci, in press.

Willmott C, Ponsford J (2009) Efficacy of methylphenidate in the rehabilitation of attention following traumatic brain injury: a randomised, crossover, double blind, placebo controlled inpatient trial. J Neurol Neurosurg Psychiatry 80:552-557.

Zirnheld PJ, Carroll CA, Kieffaber PD, O’Donnell BF, Shekhar A, Hetrick WP (2004) Haloperidol impairs learning and error-related negativity in humans. J Cogn Neurosci 16:1098-1112. 